\title{
Velocity Contour Weighting Method. I: Algorithm Development and Laboratory Testing
}

\author{
Daniel J. Howes
}

\author{
and Brett F. Sanders
}

\begin{abstract}
An algorithm is developed for real-time estimation of the cross-sectional average velocity of a channel flow by using an upwardlooking pulsed wave acoustic Doppler velocity meters (ADVM). The Velocity Contour Weighting Method (VCWM) is applicable to gradually varied flows in prismatic channels and requires little to no calibration. VCWM estimates the average velocity as a weighted average of ADVM bin velocities. Weights are based on the velocity distribution sampled by the ADVM. Collectively, the VCWM is able to adapt to a wide range of channel geometry and roughness features. Expressions for the velocity weights are developed by first applying a validated 3D computation fluid dynamics (CFD) channel flow model to a wide range of flow scenarios including differing channel geometries, discharge rates, depths, and boundary roughness. CFD simulation data are then reduced empirically with the aid of dimensional analysis to obtain the velocity weight equation. Special attention is given to the first weight accounting for near-wall velocity where the ADVM does not measure. Application of the method to a large rectangular flume shows that the VCWM predicts the average velocity with an uncertainty less than $\pm 5 \%$ and that this uncertainty can be reduced by minimizing the buffer distance between the channel bottom and the first velocity measurement. In a companion paper, the performance of the VCWM is examined in irrigation canals with trapezoidal cross sections.
\end{abstract}

\section{Introduction}

Acoustic Doppler velocity meters (ADVM) provide an alternative to traditional open channel flow measurement techniques such as stage-rating, flumes, and weirs. Installations of flumes and weirs require a significant capital investment and sufficient head (10-15\% of total depth; Replogle 1997). Head differences across the structure make the flow rating insensitive to downstream conditions and enable critical flow, two factors that support a high degree of accuracy (Chow 1959; Replogle 1997). Unfortunately, the necessary head is not always available, transitions to supercritical flow can create erosion problems, many designs trap sediment, and flumes can be difficult to configure for a wide range of flow rates and water levels (Replogle and Kruse 2007).

Pulsed ADVMs utilize acoustic transducers, which transmit an acoustic beam as a pulse of a known frequency along a narrow path (Morlock et al. 2002; Styles et al. 2006). When the pulse hits sediment or air bubbles suspended in water, it scatters and some of the sound signal returns back to the transducer. The time it takes for this "return signal" or backscatter to return to the transducer depends on the distance along the beam path at which the sediment or air bubble is located. Factors affecting the resolution of the velocity measurements include ADVM operating frequency, pulse length, fixed pulse repetition frequency, and properties of the water that affect the speed of sound such as temperature and salinity (Hardcastle and Thorne 1997). The frequency of each backscatter signal has a Doppler shift that is proportional to the fluid velocity (Morlock et al. 2002). The set of return signals therefore provides a set of distances and velocities at that moment, measured within the limited sample area of the acoustic beam.

ADVM installations in channels may utilize either side-looking configurations that sample horizontally through the cross section, or upward-looking (bottom-mounted) configurations that sample vertically through the cross section. Because of its improved accuracy in channels with variable flow depths (Styles et al. 2006), this study focuses on a pulsed, upward-looking ADVM that is mounted at the centerline of the channel and uses two velocity measurement beams.

Device software requires that information on channel geometry be input manually for the ADVM sensor to estimate discharge. Velocities are only measured by the ADVM within a small volume of the flow cross section. Therefore, in a typical cross section, an ADVM does not provide a cross-sectional average velocity, but rather a sample of the velocity distribution in a vertical plane aligned with the channel centerline. Assumptions regarding the relationship between the ADVM sample velocity and the crosssectional average velocity are typically provided within the manufacturer's software. One example is the approach presented by Huhta and Ward (2003) in which a depth integrated power-law equation was used to relate the average ADVM sample velocity 
to the cross-sectional average velocity $(V)$. However, this method has performed poorly in field applications (Styles et al. 2006).

Howes et al. (2010) describe a subcritical channel contraction design that can be used to achieve a high degree of accuracy with an upward-looking ADVM. The contraction causes rapidly varied flow that creates a relatively uniform cross-sectional velocity distribution near the contraction entrance. This makes the ADVM sample velocity a good proxy for the actual cross-sectional velocity for Froude numbers up to 0.5 . Without calibration, the crosssectional velocity can be measured within $\pm 4 \%$ for Froude numbers below 0.5 .

Although the accuracies presented in Howes et al. (2010) are considered very good for open channel flow measurement, installation of an ADVM in subcritical contraction with a Froude number below 0.5 is not always feasible and can be costly given site constraints. It should be noted that any flow measurement section including the subcritical contraction should be located in a long straight section of unobstructed flow with a consistent concrete (or equivalent) lined cross section (Styles et al. 2006).

Channel flow is typically classified as prismatic gradually varied flow (GVF) in most irrigation channels because of inline control structures. Hence, flow is not strictly uniform owing to backwater effects. Nevertheless, ADVMs are commonly deployed under these conditions, and a calibration procedure termed the index-velocity method [also referred to as the flow rate indexing procedure (QIP)] is the most common method of converting the sample velocity into the cross-sectional average velocity. The index-velocity method has been incorporated into the software run by many ADVM devices (Patino and Ockerman 1997; Morlock et al. 2002; Styles et al. 2006). The method takes the average of the sample velocities as a proxy for the true average velocity and calibrates the ADVM on the basis of site-specific attributes that are impacting the measured velocity in relation to the actual cross-sectional velocity. These attributes, the effects of which are lumped together, include channel geometry, water depth, velocities in the unmeasured "buffer" region, and boundary roughness. The primary disadvantage of the index-velocity method is that to account for all attributes, at least 10 individual calibration points at differing flow and depth conditions are recommended (Styles et al. 2006). Hence, it is time consuming, logistically challenging, and costly to implement. Moreover, estimation of the cross-sectional average velocity stands to be improved by making use of the velocity distribution, not simply the sample average.

The objective of this paper is to report a new method for estimating the cross-sectional average velocity (and discharge), in straight prismatic GVF sections, that achieves comparable accuracies to the index-velocity without calibration. The Velocity Contour Weighting Method (VCWM) presented in this study, is predicated on a weighting of the ADVM velocity measurements to obtain the cross-sectional average velocity, thus the leveraging vertical distribution of velocities provided by the ADVM. The velocity weights adapt on the basis of channel and flow properties and velocity distribution data acquired by the ADVM.

The challenge to VCWM is finding the correct weighting of velocity measurements as a function of factors that affect the velocity distribution, namely channel geometry and roughness. This is addressed by applying a validated computational fluid dynamics (CFD) model to a range of channel flow scenarios that accounts for typical geometry and roughness properties. In each case, a repeatable surrogate of the true average velocity is obtained, and it is possible to sample the ADVM velocity distribution from simulation data based on a typical instrument configuration, which is selected in this study to be $0.034 \mathrm{~m}$ bin intervals in the vertical. Moreover, CFD depicts the distribution of velocity across the entire cross section so the velocity weights can be measured with a high degree of accuracy. What remains is to understand (and predict in a reliable way) how the weights depend on channel properties. Hence, dimensional analysis and empirical modeling techniques are used for this purpose. Special attention is placed on the first weight accounting for flow near the wall because the velocity is poorly sampled near the wall, and the weight is largest and most significant relative to the cross-sectional average velocity estimate.

In this paper, VCWM development and testing in a large laboratory flume is presented. In a companion paper, the VCWM is field tested in a set of concrete-lined trapezoidal channels under a range of flow conditions.

\section{Methodology}

VCWM is a variant of the well-known velocity-area method of discharge estimation (Gupta 1989). VCWM assumes that the crosssectional area of a channel can be divided into $n$ subareas of size $A_{i}$, and for each subarea there is an average velocity $U_{i}$ so the volumetric flow rate can be computed as

$$
Q=\sum_{i=1}^{n} U_{i} A_{i}
$$

Flow rate given by Eq. (1) is readily converted to an average velocity on the basis of the wetted cross-sectional area, so VCWM can also be viewed as a nonlinear weighting of the ADVM velocities

$$
V_{\mathrm{VCWM}}=\frac{Q_{\mathrm{VCWM}}}{A}=\sum_{i=1}^{n} w_{i} U_{i}
$$

where $w_{i}=A_{i} / A$ represents weights that sum to unity and $U_{i}$ $i=2, \ldots, n$, represents monotonically increasing ADVM measurements of velocity, whereas $U_{1}$ represents the average velocity in the so-called buffer distance $\left(z_{b}\right)$ near the ADVM sensor.

The areas associated with each velocity measurement, $A_{i}$, $i=1, \ldots, n-1$ are taken as the cross-sectional area between two neighboring contour values, $u_{i}$ and $u_{i+1}$. $A_{n}$, represents the cross-sectional area associated with a velocity greater than $u_{n}$. The contour values are set as $u_{i}=\left(U_{i-1}+U_{i}\right) / 2$ for $i=3, \ldots, n$, which represents the average of neighboring velocity measurements. The first contour $u_{1}=0$ because this corresponds to the channel boundary, and a special procedure is used to define $u_{2}$ because it is only bounded by ADVM measurements from above. In this case, an extrapolation procedure is used: $u_{2}=2 U_{2}-U_{3}$. When the bin locations are evenly spaced (above the buffer), the vertical height of the contours along the channel centerline is also evenly distributed because a linear average of neighboring velocities defines the contour values (Fig. 1).

The linkage between the weights and the velocity distribution inherent to VCWM is intriguing because it raises the possibility that by observing the vertical distribution of velocity, in light of the cross-sectional area and roughness of the channel, one can obtain both the velocity and weight values required by Eq. (2), improve the accuracy of discharge estimates, and possibly eliminate calibration requirements compared with the index-velocity method.

The buffer distance, $z_{b}$, refers to the distance between the channel bottom and the first velocity measurement for upward-looking ADVM deployments (Fig. 1). This is affected by two factors: the ADVM device height, which depends on the mounting configuration and the instrument blanking distance. The latter corresponds to a region above the sensor where no measurements are taken so the ADVM transducer has time to switch from transmitter (sending 


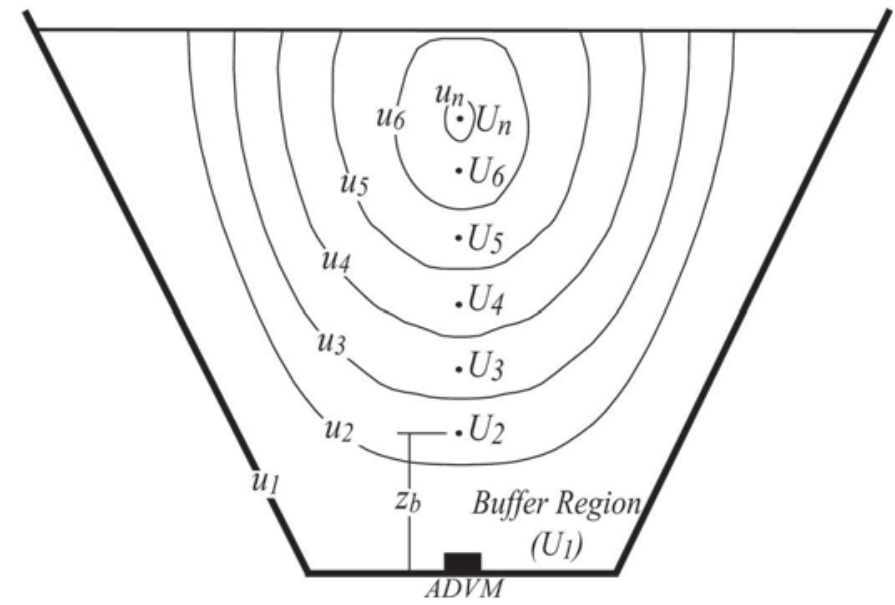

Fig. 1. Conceptual velocity contours in a prismatic trapezoidal channel showing the location of velocity measurements $\left(U_{i}\right)$ in relation to the ADVM at the bottom of the channel; the contour area, $A_{i}$, is the area within contours $u_{i}$ and $u_{i+1}$

the pulse) to receiver, where it begins receiving the backscatter signal (Simpson 2001). The ADVM itself disrupts the local velocity distribution so the buffer typically ensures that the first measurement is outside the affected region. Typical values of $z_{b}$ range from 0.14 to $0.2 \mathrm{~m}$.

The absence of near-bottom velocity measurements poses a challenge to discharge measurement with upward-looking ADVM devices. Indeed, Fig. 1 shows that a significant fraction of the channel cross section is associated with near-wall velocities. As described subsequently, a strategy to interpolate velocity in the buffer region based on ADVM measurements is developed as an integral part of the proposed VCWM.

\section{Computational Fluid Dynamics Modeling}

The three-dimensional (3D) computational fluid dynamics (CFD) model, Flow 3D (Flow Science Inc., Santa Fe, NM) was utilized in this study to simulate flow in a range of scenarios characterized by varying flow rate, surface roughness, and channel geometries. In each case, the weights, $w_{i}$, required by the VCWM were computed from CFD model output. CFD is ideal for this purpose because it supports a high level of control (discharge is known exactly), a complete characterization of the velocity distribution, and can readily account for different channel configurations.

Flow 3D solves the Reynolds-Averaged Navier-Stokes (RANS) equations by finite-volume method, three dimensionally by using a Cartesian grid system, achieves turbulent closure using Renormalization-Group (RNG) turbulence model, and resolves free surface flow by using a volume-of-fluid (VOF) model (Hirt and Nichols 1981). Flow 3D handles free surface flows well and has been field tested under a wide range of hydraulic conditions (Cook and Richmond 2001).

A detailed description of the CFD model setup and calibration used for this study can be found in Howes et al. (2010), but a brief summary is presented in this study. The CFD model was configured for a rectangular channel of the same dimensions as a large rectangular flume at the Irrigation Training and Research Center (ITRC) at California Polytechnic State University, San Luis Obispo (Cal Poly flume). The painted steel Cal Poly ITRC flume has dimensions of $1.215 \mathrm{~m}$ in width by $1.215 \mathrm{~m}$ in depth by $86 \mathrm{~m}$ in length with a bottom slope of 0.002 . The testing region of the Cal Poly flume and the CFD model flume are approximately $54 \mathrm{~m}$ long.
At the downstream end of the flume, a vertical weir is used to fix the water level in the testing region. The CFD model was calibrated by adjusting the surface roughness within a realistic range of values for the flume walls and validated by comparison with a different set of flow conditions.

Cross-sectional velocity samples were measured in the Cal Poly ITRC flume under different flow rate, water depth, and turbulence scenarios. An evenly spaced grid of 36 velocity samples were measured with a SonTek/YSI FlowTracker Handheld-ADV (acoustic Doppler velocimeter). These velocity measurements were compared with the velocities extracted from the same location in the CFD model flume using the same flow and depth scenarios. Results of the validation procedure showed the coefficient of variation of the root mean squared error [CVRMSE is the root mean squared error (RMSE) of the 36 velocities divided by the actual crosssectional average velocity at the measurement location] of 4 and $8 \%$ under 10 flow scenarios. This error can be partially attributed to the inability of any CFD to completely resolve turbulence at scales that are computationally feasible, and unlike the CFD simulated flume, the sides and bottom of the Cal Poly ITRC flume are not perfectly flat so some variation in velocity between the CFD flume and the physical flume are expected. The results of the validation were deemed sufficient to proceed with the simulation needed to develop the Velocity Contour Weighting Method.

For this study, the validated Flow 3D model was applied to simulate channel flow scenarios involving multiple discharge rates, flow depths, channel geometries, and channel roughnesses. Three channel geometries were modeled: a rectangular $(\mathrm{R})$ section with a bottom width $(B)$ of $1.22 \mathrm{~m}$; a trapezoidal channel (T1) with a $B$ of $0.61 \mathrm{~m}$ and a side slope $(S S)$ of 0.51 ( $0.51: 1$, horizontal:vertical); and a trapezoidal channel (T2) with a $B$ of $1.5 \mathrm{~m}$ and $S S$ of 1.5 . Each channel was modeled under four flow rate scenarios of 0.283 , $0.425,0.566$, and $0.708 \mathrm{~m}^{3} / \mathrm{s}$ and each flow was modeled under two depths nominally 1 and $0.65 \mathrm{~m}$. The depth was adjusted by using a weir at the downstream end of the modeled channel. In addition, each of these scenarios was modeled under three roughnesses $\left(k_{s}\right)$ equivalent to smooth steel $\left(k_{s}=0.0002 \mathrm{~m}\right)$, finished concrete $\left(k_{s}=0.0015 \mathrm{~m}\right)$, and bare earth $\left(k_{s}=0.012 \mathrm{~m}\right)$ for a total of 72 individual model scenarios.

The deployment of an ADVM was simulated by sampling the streamwise velocity, $U_{i}$, along a $1 \mathrm{D}$, vertically aligned column of fluid located at the center of the channel. The sample velocities were evenly spaced at $0.034-\mathrm{m}$ intervals from just above the channel bottom to just below the water surface. An ADVM object was not incorporated into the CFD channel geometry. Although the object, mounted on the channel bottom, would affect the velocity distribution, ADVM devices are available in different sizes and geometries. In addition, many possible mounting locations exist, above or below the channel bottom. It was beyond the scope of this study to examine multiple upward-looking ADVM devices and mounting configurations. As previously described, device blanking distance (distance from ADVM body to the first measurement) should be large enough so that the first measurement is taken outside of any localized flow disturbance.

For each scenario, the 2D, cross-sectional distribution of streamwise velocity was exported onto a Cartesian grid and a histogram of the grid values, using hypothetical contour values $u_{i}$ as histogram edges, was computed. That is, the number of grid cells with a value between neighboring contour values were counted. The value $A_{i}$, from Eq. (1) and (2), was computed as the number of grid cells or pixels that were bounded by the $u_{i}$ and $u_{i+1}$ contours, multiplied by the area of each grid cell. Histogram analysis requires that $U_{i}$ are monotonically increasing, although this is not always the case because the velocity maximum is submerged below the free surface 
(dip-phenomenon). Hence, only those bins that fall at or below the velocity maximum are considered. This does not restrict the VCWM from a full accounting of the channel cross section, in particular the near-surface velocities, because these regions are contained within contours defined by velocity bins below the velocity maximum as shown in Fig. 1.

\section{Weighting Function}

Once the $A_{i}$ values were computed from the histogram analysis, $w_{i}$ was computed as $w_{i}=A_{i} / A$ where $A$ is the cross-sectional wetted area. Fig. 2 shows an example of the weights for two of the 72 scenarios considered. Notice that the weights decrease with height, which indicates that the contour areas are decreasing in size from the channel bottom to the maximum velocity point as shown in Fig. 1.

The remaining challenge is to develop an empirical model for the vertical distribution of weights that is sensitive to the range of channel geometries, surface properties, and flow conditions relevant to irrigational channel flow measurement. From dimensional analysis, the weights are expected to depend on several factors as follows:

$$
w_{i}=f\left(\frac{z_{i}}{z_{U \max }}, \frac{\Delta z}{z_{U \max }}, \frac{k_{s}}{z_{U \max }}, \mathrm{R}, R_{S}\right)
$$

where $z_{i}=$ height of measurement $U_{i}$ associated with the weight, $w_{i}$, relative to the channel bottom; $z_{U \max }=$ height of the highest velocity within the sample profile; $\Delta z=$ vertical distance between ADVM velocity samples; $k_{s}=$ equivalent roughness; $\mathrm{R}=$ Reynolds number; and $R_{S}=$ channel shape factor. The characteristic length scale is $z_{U \text { max }}$ because this brackets ADVM measurements that are incorporated into the VCWM and also characterizes the thickness of the bottom boundary layer. The five dimensionless groups in Eq. (3), from left to right, account for the height of the velocity measurement, the density of velocity measurements, roughness effects, Reynolds number effects, and shape effects. Through a trial-and-error process, it was determined that the weights could be characterized most effectively by using only the first two dimensionless groups as follows:

$$
w_{i}=1.78\left(\frac{\Delta z\left(z_{U \max }-z_{i}\right)}{z_{U \max }^{2}}\right)
$$

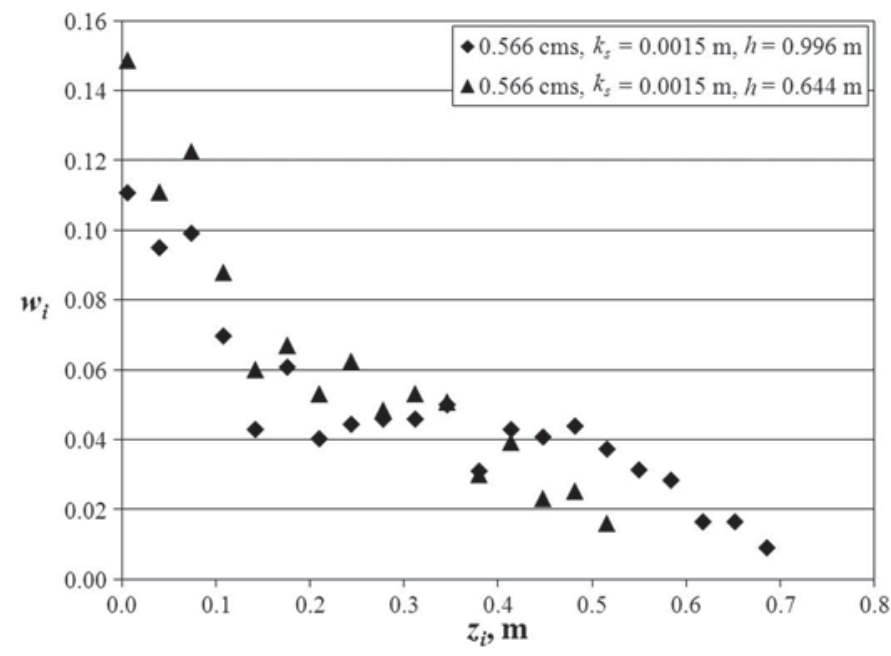

Fig. 2. Weights by height from channel bottom to the maximum velocity height for two scenarios from the rectangular channel simulations with the same roughness and flow rates but different depths
Fig. 3(a) shows the linear fitting of Eq. (4) $\left(R^{2}=0.845\right)$ based on all 72 simulations. A third-order polynomial regression was also investigated as a possible weight function [Fig. 3(b)]. The $R^{2}$ was improved to 0.90 , but the polynomial equation did not show any improvement over Eq. (4) in discharge measurement accuracy during laboratory testing. Therefore, the simpler equation [Eq. (4)] was selected. The fact that an effective fitting of the weights was achieved with only two of the dimensionless groups is surprising considering that channel shape, Reynolds number, and roughness all contribute significantly to the velocity distribution in open channels. The interpretation in this work of this result is that these effects are directly captured by a combination of the velocity measurements and the boundary layer thickness that is chosen as the characteristic length scale.

At least a portion of the variability in weight shown in Fig. 3 is owing to the relatively coarse grid of the CFD velocities used for the histogram analysis. An analysis was conducted for one of the scenarios by increasing the grid resolution $50 \%$. This had a smoothing effect on the weight distribution, however the trend of the weight values did not change. It was computationally infeasible to utilize the finer grid spacing for all 72 scenarios. Had a finer grid been used, Eq. (4) would have likely been the same; however, the $R^{2}$ value may have been higher.

The sum of $w_{i}$ in a cross section should equal unity (1). To ensure unity, $w_{i}$ should be computed for each of the measurement
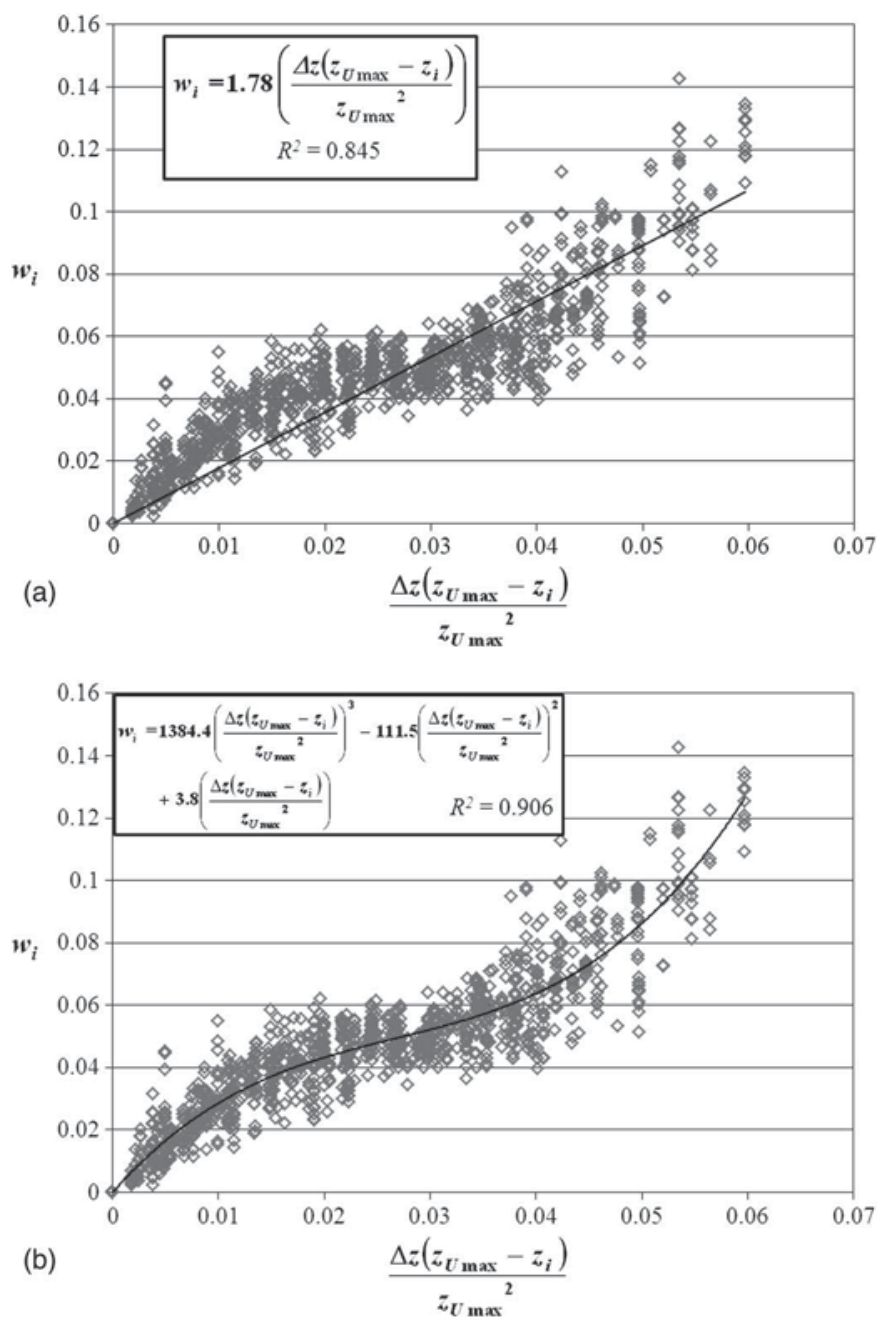

Fig. 3. Weights developed from the histogram analysis related to $z_{i}$, $\Delta z$, and $z_{U \max }$ with (a) linear fitting; and (b) polynomial fitting 
locations, from first measurement at $z_{b}\left(U_{2}\right)$ to the measurement at the $z_{U \max }\left(U_{\max }\right)$, and the buffer region's weight should be computed as

$$
w_{1}=1-\sum_{i=2}^{n} w_{i}
$$

Combining Eqs. (1), (4), and (5), the cross-sectional average velocity can be computed as

$$
V_{\mathrm{VCWM}}=\sum_{i=2}^{n} w_{i} U_{i}+\left(1-\sum_{i=2}^{n} w_{i}\right) U_{1}
$$

where $V_{\mathrm{VCWM}}=$ computed cross-sectional average velocity and the sum of the weights are computed from $2<i<n$ by using Eq. (4). The location $i=2$ coincides with the first measurement from the ADVM and location $n=$ maximum velocity point. The second term on the right side of Eq. (6) is the buffer region weight and estimated velocity $\left(U_{1}\right)$.

\section{Estimating Velocity in the Buffer Region}

From the 72 CFD simulations, buffer region weight $\left(w_{1}\right)$ ranged from 0.3 to 0.5 varying by water depth and channel geometry (assuming a buffer height of $0.14 \mathrm{~m}$ ). The large weight of this unmeasured region illustrates the need for an accurate estimate of $U_{1}$.

Power and logarithmic velocity distributions are commonly used to describe velocity profiles in open channel flow. These relationships are applicable to well-developed boundary layers, where the velocity distribution is monotonically increasing from the boundary to the region of interest (Maghrebi and Rahimpour 2005). Because the $A_{1}$ is near the boundary, it can be assumed that in fact the velocities in the region will be monotonically increasing. The power-law velocity distribution for wide shallow uniform channel flow can be represented as (Chen 1991)

$$
\frac{u}{u_{*}}=c\left(\frac{z}{z^{\prime}}\right)^{1 / m}
$$

where $u=$ velocity at height $z ; u_{*}=$ shear velocity; $z^{\prime}=$ characteristic length, estimated for turbulent flows as the surface roughness height $\left(k_{s}\right)$ divided by $30 ; c=$ power-law coefficient; and $1 / m$ is the power-law exponent. Previous researchers have determined $m$ typically varies between 4 and 12 depending on Reynolds number for hydraulically smooth flow and roughness for fully turbulent flow (Chen 1991). The coefficient $c$ can be shown to be proportional to $m$. A number of studies have suggested that an $m$ of 6-7 is valid for many wide shallow open channel flow situations (Chen 1991; Cheng 2007). However, in many open channel flow measurement situations, the assumption of wide shallow uniform flow is not valid. The channel banks impact the velocity distribution and in most cases uniform flow does not exist because of control structures downstream of flow measurement sections. In addition, simply assuming a constant exponent over a range of flow situations, including fluctuating depths and velocities, would likely lead to increased measurement error.

The centerline velocity data from CFD analyses of all 72 scenarios were used to develop a method of computing the power-law coefficient and exponent on the basis of measurable data and flowmeasurement site constraints, specifically a stable prismatic cross section with constant roughness. Centerline velocity values from just above the channel bottom to $0.244 \mathrm{~m}$ in depth were considered. Because it can be assumed that the ADVM measured velocities are related to the cross-sectional average velocity, and the average of
ADVM measured velocities can be measured, a modified version of the power-law was used as shown in Eq. (8)

$$
\frac{u}{V_{\mathrm{ADVM}}}=a\left(\frac{z}{h}\right)^{1 / m}
$$

where $u=$ velocity at height $z$ between the channel bottom and the first measurement, $U_{2} ; V_{\mathrm{ADVM}}=$ average of the measured velocities from $U_{2}$ to just below the water surface $\left(U_{n}\right) ; h=$ flow depth; $a=$ modified power-law coefficient; and $1 / \mathrm{m}=$ power-law exponent.

Examining $a$ and $m$ in Eq. (8) with the CFD modeled velocities from just above the channel bottom to $0.244 \mathrm{~m}$, both variables showed strong relationships to the $k_{s}$ and $R_{h}$, which is consistent with other studies (Chen 1991; Cheng 2007). $V_{\text {ADVM }}$ varies by the ADVM buffer height, therefore $a$ should also have some variability. For a $z_{b}$ of $0.142 \mathrm{~m}$, the coefficient $a$ varied between 1.019 and 1.105 over the scenarios tested, whereas the exponent $m$ varied between 6.26 and 13.80. An $m$ above 12 is unrealistic for most real-world situations and is likely a result of the very low surface roughness used in the model (for painted steel) in conjunction with the rectangular channel geometry. Several studies have indicated relationships between $c$ and $m$ in Eq. (7) (Chen 1991). From the simulated data, the linear relationship found between $a$ and $m$ in Eq. (8) with a $z_{b}$ of $0.142 \mathrm{~m}$ is shown in Fig. 4.

The same methodology shown in Fig. 4 was used with $z_{b}$ values of $0.074,0.108,0.142,0.176,0.210$, and $0.244 \mathrm{~m}$. Under each scenario, $m$ remained unchanged, whereas $a$ showed some variability. The slope in the linear relationship, from Fig. 4 varied although the $y$ intercept, 1 , remained constant. The general linear relationship for $a$ related to $m$ can be shown as

$$
a=\left(C_{a} m\right)^{-1}+1
$$

where $C_{a}=$ coefficient derived as a function of the ratio $z_{b}$ and the maximum recommended buffer distance, $z_{b \max }\left(z_{b \max }=0.25 \mathrm{~m}\right)$ used to develop this relationship. The relationship between $C_{a}$ and the ratio of $z_{b}$ to $z_{b \max }$ is shown in Fig. 5 and as $\left(R^{2}=0.994\right)$

$$
C_{a}=1.65\left(\frac{z_{b}}{z_{b \max }}\right)+0.9
$$

The inverse power law exponent, $m$, developed by using the centerline velocities from the CFD scenarios, varies by channel geometry and boundary roughness. In wide rectangular channels,

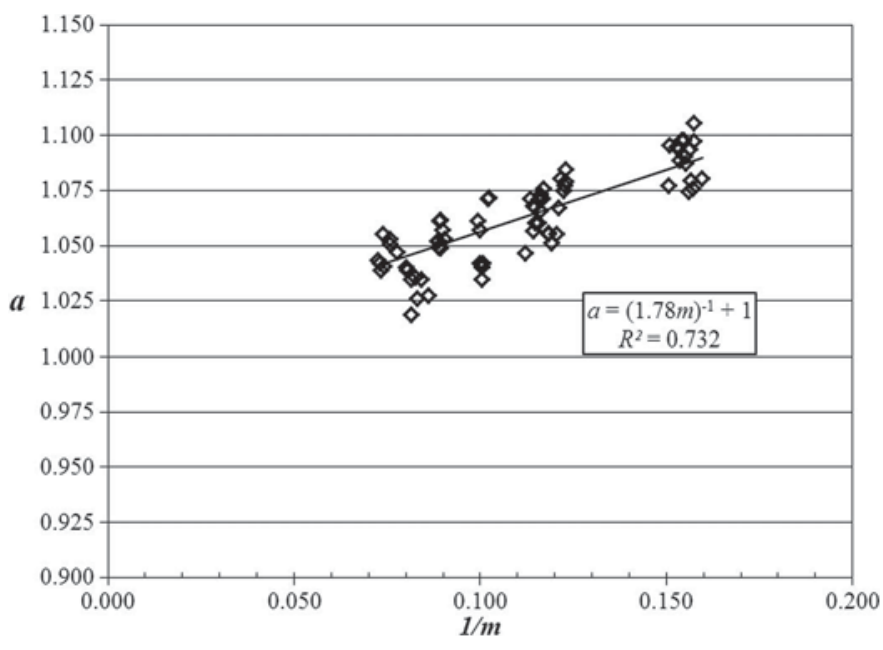

Fig. 4. Relationship between Eq. (8) power-law coefficient and exponent for $z_{b}=0.142 \mathrm{~m}$ 


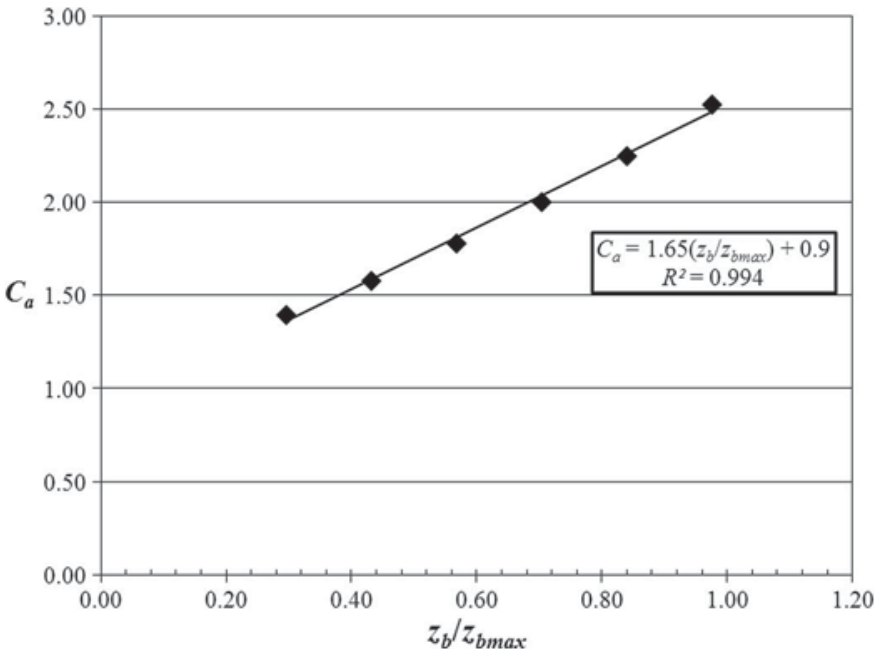

Fig. 5. Relationship between the coefficient $C_{a}$ and ADVM buffer distance, $z_{b}$

the relationship between $m$ and the Darcy friction factor, $f$, is (Chen 1991)

$$
m=\frac{\kappa}{\sqrt{f / 8}}
$$

where $\kappa$ is von Kármán constant $(\kappa=0.4)$. For fully rough flows, the Darcy friction factor can be computed on the basis of $R_{h}$ and $k_{s}$ as (Keulegan 1938)

$$
\frac{1}{\sqrt{f}}=-2.03 \log \left(\frac{k_{s}}{12.2 R_{h}}\right)
$$

Substitution of Eq. (12) into Eq. (11) gives a model for $m$ that was fit to the combined CFD data shown in Fig. 6(a) to obtain the following expression $\left(R^{2}=0.660\right)$ :

$$
m=1.17\left[-2.03 \kappa \sqrt{8} \log \left(\frac{k_{s}}{12.2 R_{h}}\right)\right]
$$

Fig. 6(a) shows the fit for individual channel side slopes. Eq. (13) is nearly identical to the fit for the $S S=0.5$. However, this expression does not capture all of the variability in the data because the slopes of the fitting equations for the rectangular channel $(S S=0)$ and the trapezoidal channel with an $S S=1.5$ are 1.38 and 1.0, respectively. Interestingly, the CFD data for the trapezoidal channel with a $S S=1.5$ has a one-to-one relationship with the formula Keulegan (1938) derived for trapezoidal channels. As shown in Fig. 6(b), by introducing an additional shape factor which accounted for the variable side slope, the following improved model was obtained $\left(R^{2}=0.968\right)$ :

$$
m=\left(\frac{1.17}{(S S+0.5)^{0.24}}\right)\left[-2.03 \kappa \sqrt{8} \log \left(\frac{k_{s}}{12.2 R_{h}}\right)\right]
$$

The shape factor on the left side of Eq. (14) becomes approximately 1.0 when the side slope is 1.5 , which is in agreement with Keulegan's formula for trapezoidal channels. An interesting factor with regard to Fig. 6 is that even though $R_{h}$ incorporates channel shape, the inclusion of the channel shape factor shows a significant improvement. This would indicate that channel side slope has a major influence on velocity distribution and is not fully accounted for in the $R_{h}$ term, which is consistent with other research findings in which rectangular, trapezoidal, triangular, and semicircular
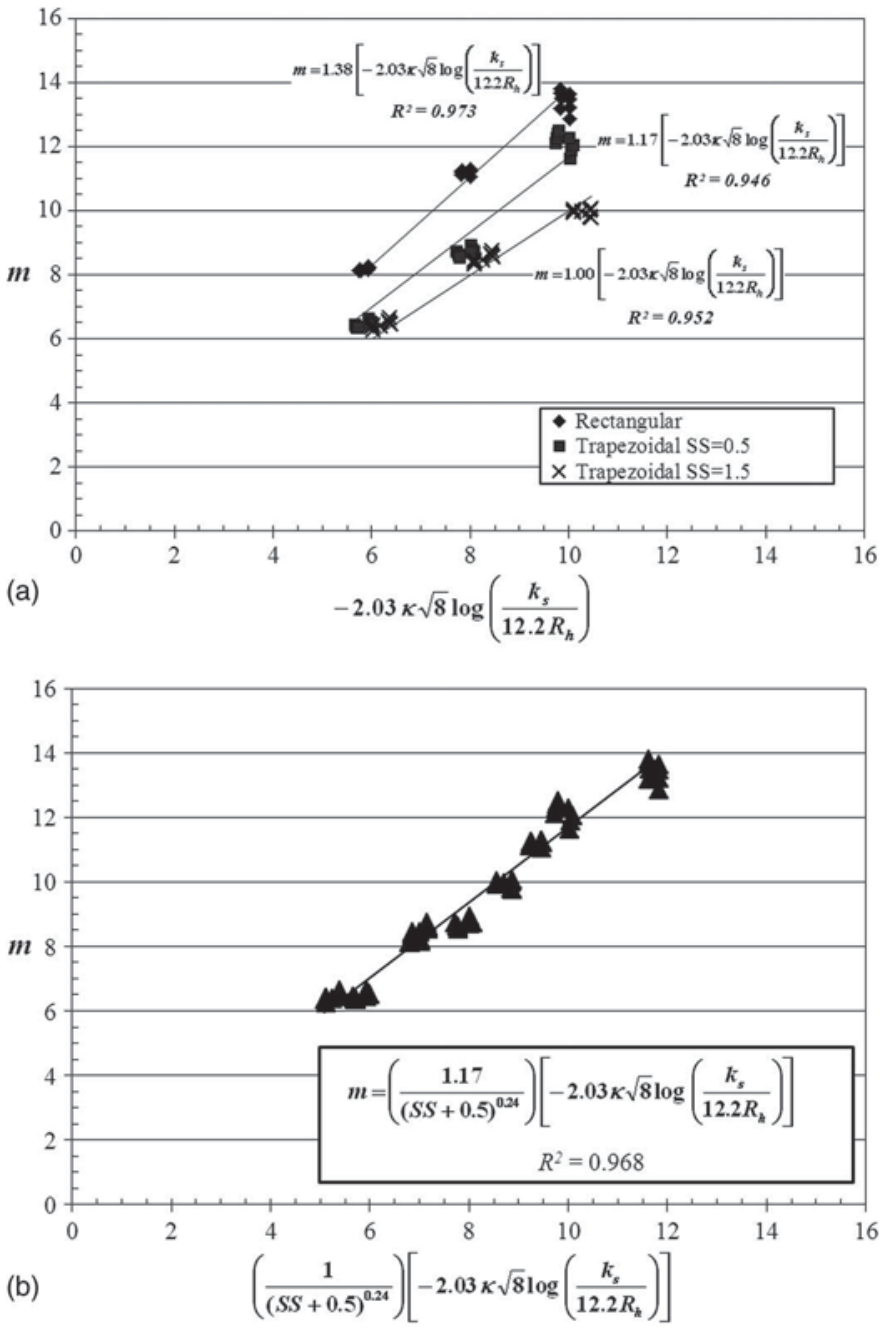

Fig. 6. The inverse power-law exponent, $m$, related to a function of $R_{h}$, and $k_{s}$ : (a) without channel shape factor; and (b) with channel shape factor

channels were compared (ASCE 1963; Keulegan 1938; Montes 1998). It is important to point out that much of the research on power-law velocity distributions, including the relationship between $m$ and $f$ shown in Eq. (11), has been conducted assuming wide, shallow, open channel flow. It should not be surprising that some inclusion of $S S$ is necessary for narrower channels, such as the ones examined in this study. Furthermore, most channels found in irrigation and drainage projects would not be considered wide and shallow.

The $m$ values computed by using Eq. (14) should be limited to 12 for realistic physical situations. Situations in which the channel boundary conditions are very smooth, for example, new painted steel, $m$ values approaching or equal to 12 are possible depending on channel geometry. In most ADVM applications, values for $m$ will be less than this value. Combining Eq. (8) with the relationship between $a$ and $m$ shown in Eq. (9), the velocities in the buffer region of the channel can be estimated at discrete heights, $z$, by

$$
\frac{u}{V_{\mathrm{ADVM}}}=\left[\left(C_{a} m\right)^{-1}+1\right]\left(\frac{z}{h}\right)^{1 / m}
$$

where $m$ is computed by using Eq. (14) and $C_{a}$ is computed from Eq. (10). The average centerline streamwise velocity between the channel bottom and the first contour boundary (i.e., buffer region 
average velocity, $\left.U_{1}\right)$ is required for Eq. (6). The buffer region average velocity $\left(U_{1}\right)$ can be estimated by integrating Eq. (15) over the heights $z=0$ to $z=z_{b}^{\prime}$ as

$$
\frac{U_{1}}{V_{\mathrm{ADVM}}}=\frac{1}{\left(z_{b}^{\prime}-0\right)} \int_{0}^{z_{b}^{\prime}}\left[\left(C_{a} m\right)^{-1}+1\right]\left(\frac{z}{h}\right)^{1 / m} d z
$$

where $z_{b}^{\prime}=$ vertical distance from the channel bottom to the first contour boundary at the centerline. The first measured velocity, $U_{2}$, measured at $z_{b}$, is located between the second and third contour boundaries, $u_{2}$ and $u_{3}$ as shown in Fig. 1 (first contour boundary $u_{1}$ is the channel bottom and sides). Contour boundaries identified by velocities $u_{2}$ and $u_{3}$ are separated by approximately $0.034 \mathrm{~m}$ vertically at the centerline with $U_{2}$ located in the center. Therefore, the height of the second contour boundary, $z_{b}^{\prime}$, is equal to $z_{b}$ minus half the distance between the contour boundaries $\left[z_{b}^{\prime}=z_{b}-0.5\right.$ $(0.034 \mathrm{~m})]$ at the channel centerline. The resulting equation that can be used to estimate $U_{1}$ is

$$
\frac{U_{1}}{V_{\mathrm{ADVM}}}=\left[\frac{\left(C_{a} m+1\right) z_{b}^{\prime / m}}{C_{a} h^{1 / m}(m+1)}\right]
$$

\section{Laboratory Testing}

An open flume at the Irrigation Training and Research Center (ITRC), California Polytechnic State University, San Luis Obispo (Cal Poly), was used to evaluate the VCWM in a physical application. The rectangular Cal Poly ITRC flume has dimensions of $1.215 \mathrm{~m}$ in width, $1.215 \mathrm{~m}$ in depth, and $86 \mathrm{~m}$ in length with a bottom slope of 0.002 . Flume components are capable of handling flow rates up to $0.85 \mathrm{~m}^{3} / \mathrm{s}$. The testing region of the flume is approximately $54 \mathrm{~m}$ long. The start point of the testing region was just downstream of a flow conditioner consisting of a 1-m-long honeycomb of 0.076-m diameter PVC pipes. At the downstream end of the flume, a vertical weir is used to fix the water level in the testing region.

A recirculation facility is utilized at the Cal Poly flume during evaluations. The discharge is measured in real time by a calibrated 0.76-m diameter McCrometer Magmeter (Hemet, CA.) installed in a long, straight section of pipe feeding the head of the flume. The discharge into the flume is regulated through a valve at the flume entrance. The Magmeter has been calibrated, by using a weigh tank, to within $\pm 2 \%$ of the actual flow.
A Sontek/YSI Inc., (San Diego, CA.) Argonaut SW (SonTek $\mathrm{SW}$ ), an upward-looking ADVM with a beam angle of $45^{\circ}$, was installed within the flume's flow measurement section. Velocity profile data from the SonTek SW was extracted at 0.034-m intervals starting at the buffer distance $\left(z_{b}\right)$ to the closest interval below the water surface. Velocities at each depth were averaged over 5-min intervals and recorded by the ADVM. The number of 5-min average samples for each testing scenario ranged from 14-23 readings.

Tests were conducted under nominal flow rates of 0.425 and $0.566 \mathrm{~m}^{3} / \mathrm{s}$ and at two flow depths nominally of 0.65 and $0.95 \mathrm{~m}$. Three buffer distances $\left(z_{b}\right)$ were examined under each of the four scenarios, $0.144,0.178$, and $0.212 \mathrm{~m}$. Eq. (6) was used to compute $V_{\mathrm{VCWM}}$ utilizing the individual velocities measured by the SonTek SW and where $w_{i}$ was computed with Eq. (4) and (5) with $U_{1}$ computed with Eq. (17).

The actual cross-sectional velocity $(V)$ was calculated based on the discharge from the Magmeter, SonTek SW water level, and channel width. A staff gauge installed at the testing section was used as a check insuring the SonTek SW was measuring water depth correctly. Flow rates were sampled by the Magmeter every $2 \mathrm{~s}$ and averaged over the same 5-min period as the SonTek SW. The relative error (percentage) between $V_{\mathrm{VCWM}}$ and $V$ was computed by using Eq. (18)

$$
\text { Relative error }=\frac{\left(V_{\mathrm{VCWM}}-V\right)}{V} \times 100
$$

The results of the laboratory testing of the VCWM algorithm in the Cal Poly ITRC flume are shown in Table 1. Because the flume is clean, painted steel and rectangular in shape, the low roughness and channel geometry resulted in a computed value of $m$ [Eq. (12)] greater than 12 . Therefore, for these testing scenarios, the $m$ was set at the maximum limit of 12. The Froude number, F, shown in Table 1 was computed using the actual cross-sectional velocity and water depth measured by the ADVM.

For each of the flow test scenarios, the mean relative error of the computed cross-sectional velocity $\left(V_{\mathrm{VCWM}}\right)$ are shown and also the upper and lower confidence limits of the error within the sample population based on $99 \%$ confidence limits. The mean relative error ranged from -0.01 to $2.00 \%, 1.07-2.69 \%$, and $2.64-3.50 \%$ for $z_{b}$ equal to $0.144,0.178$, and $0.212 \mathrm{~m}$, respectively. The $99 \%$ confidence limits ranged from -0.81 to $3.39 \%, 0.62-4.06 \%$, and $1.36-4.75 \%$ for $z_{b}$ equal to $0.144,0.178$, and $0.212 \mathrm{~m}$,

\begin{tabular}{|c|c|c|c|c|c|c|c|c|c|c|}
\hline \multirow{2}{*}{$\begin{array}{l}\text { Average } \\
\text { discharge } \\
\left(\mathrm{m}^{3} / \mathrm{s}\right)\end{array}$} & \multirow[b]{2}{*}{$\begin{array}{l}z_{b} \\
(\mathrm{~m})\end{array}$} & \multirow[b]{2}{*}{$\begin{array}{c}\text { Number of } \\
\text { 5-m samples }\end{array}$} & \multicolumn{5}{|c|}{ Average } & \multirow{2}{*}{$\begin{array}{l}\text { Mean relative } \\
\text { error of } V_{\mathrm{VCWM}} \\
(\%)\end{array}$} & \multicolumn{2}{|c|}{$99 \%$ confidence interval of erro } \\
\hline & & & $\begin{array}{l}\text { Depth } \\
\text { (m) }\end{array}$ & $F$ & $m$ & $\begin{array}{c}V_{\mathrm{VCWM}} \\
(\mathrm{m} / \mathrm{s})\end{array}$ & $\begin{array}{c}V \\
(\mathrm{~m} / \mathrm{s})\end{array}$ & & $\begin{array}{l}\text { Upper } \\
(\%)\end{array}$ & $\begin{array}{l}\text { Lower } \\
(\%)\end{array}$ \\
\hline 0.440 & 0.144 & 23 & 0.618 & 0.24 & 12 & 0.587 & 0.585 & $0.30 \%$ & $1.10 \%$ & $-0.51 \%$ \\
\hline 0.440 & 0.144 & 14 & 0.927 & 0.13 & 12 & 0.400 & 0.392 & $2.00 \%$ & $3.39 \%$ & $0.61 \%$ \\
\hline 0.570 & 0.144 & 18 & 0.642 & 0.29 & 12 & 0.731 & 0.731 & $-0.01 \%$ & $0.78 \%$ & $-0.81 \%$ \\
\hline 0.570 & 0.144 & 14 & 0.921 & 0.17 & 12 & 0.515 & 0.510 & $1.07 \%$ & $2.28 \%$ & $-0.14 \%$ \\
\hline 0.440 & 0.178 & 23 & 0.618 & 0.24 & 12 & 0.594 & 0.585 & $1.47 \%$ & $2.32 \%$ & $0.62 \%$ \\
\hline 0.440 & 0.178 & 14 & 0.927 & 0.13 & 12 & 0.403 & 0.392 & $2.69 \%$ & $4.06 \%$ & $1.32 \%$ \\
\hline 0.570 & 0.178 & 18 & 0.642 & 0.29 & 12 & 0.741 & 0.731 & $1.39 \%$ & $2.15 \%$ & $0.63 \%$ \\
\hline 0.570 & 0.178 & 14 & 0.921 & 0.17 & 12 & 0.520 & 0.510 & $1.92 \%$ & $3.18 \%$ & $0.66 \%$ \\
\hline 0.440 & 0.212 & 23 & 0.618 & 0.24 & 12 & 0.601 & 0.585 & $2.74 \%$ & $3.54 \%$ & $1.93 \%$ \\
\hline 0.440 & 0.212 & 14 & 0.927 & 0.13 & 12 & 0.406 & 0.392 & $3.50 \%$ & $4.75 \%$ & $2.25 \%$ \\
\hline 0.570 & 0.212 & 18 & 0.642 & 0.29 & 12 & 0.751 & 0.731 & $2.67 \%$ & $3.38 \%$ & $1.96 \%$ \\
\hline 0.570 & 0.212 & 14 & 0.921 & 0.17 & 12 & 0.523 & 0.510 & $2.64 \%$ & $3.92 \%$ & $1.36 \%$ \\
\hline
\end{tabular}
respectively.

Table 1. Results of Laboratory Testing of the VCWM by Using an Upward-Looking ADVM for $z_{b}=0.144,0.178$, and $0.212 \mathrm{~m}$ 


\section{Discussion}

The VCWM is comprised of two components, the weighting algorithm and a method to estimate the velocity in the buffer region $\left(U_{1}\right)$. These two components are computed independently, meaning if centerline velocities from an upward-looking ADVM could be measured from the channel bottom to the first velocity reading below the water surface (i.e., no buffer distance), no need would exist to estimate $U_{1}$.

On the basis of dimensional analysis [Eq. (3)], it was expected that the weights could depend on the relative measurement height, relative bin size, relative roughness, Reynolds number, and shape effects. However, only the first two of these parameters were found to be significant [Eq. (4)]. Conversely, relative roughness and channel shape proved important for characterizing the near-wall velocity $U_{1}$, effectively filling the gap in the measured velocity distribution. These results suggest that the VCWM implicitly accounts for roughness, Reynolds number, and shape effects by directly measuring the velocity distribution. The weights need only account for details of the velocity measurements, such as height and spacing. Moreover, information about roughness, channel shape, and Reynolds number is only needed in the case of a gap in the measured velocity distribution. The companion paper will examine this hypothesis further by applying the VCWM sensitivity to a number of field sites with range of $k_{s}$ values for concrete-lined channels.

As noted previously, the vertical sampling spacing proved to be an important factor. The $\Delta z$ used for the VCWM development was $0.034 \mathrm{~m}$. This is the same nominal vertical sampling distance utilized by the ADVM used in the laboratory testing. However, $\Delta z$ is a function of the speed of sound in water, which is influenced by water temperature and quality; however, typically remains within $\pm 0.001 \mathrm{~m}$ of nominal. The $\Delta z$ computed by the ADVM should be used in Eq. (4). A benefit of including the $\Delta z$ in the VCWM is that if future devices are developed with different $\Delta z$, it should be possible to utilize the VCWM for these devices. The CFD model was utilized to evaluate three scenarios in which $\Delta z=0.01,0.034$, and $0.05 \mathrm{~m}$. The VCWM estimated cross-sectional velocities from the three $\Delta z$ were compared and the values were within $2 \%$ of one another. This brief evaluation indicates that the VCWM can be used for devices with different $\Delta z$; however, this should be verified through laboratory or field testing once a device has been developed.

Mean relative percentage error results in Table 1 show some bias toward over estimation of the cross-sectional average velocity under the conditions analyzed in the laboratory. The first issue that could contribute to this result is related to the laboratory flume being very smooth and rectangular. The testing was conducted at one extreme of the scenarios utilized to develop the relationships for the VCWM. Another variable that impacts $V_{\mathrm{VCWM}}$ is the buffer height, $z_{b}$. At the higher buffer height of $0.212 \mathrm{~m}$, the error is the most significant. This demonstrates the importance of maximizing the measurement region, thereby decreasing the weight $\left(w_{1}\right)$ of the buffer region. A smaller buffer region reduces the significance of the estimated buffer region velocity. However, care must be taken with a smaller blanking distance so that measurements are taken only outside of any flow field disturbance caused by an ADVM mounted in the flow path.

A theoretical ADVM was not included as an object in the CFD modeling. A potential concern is related to the possible flow disturbance caused by the device projecting into the flow path and the effect it would have on the VCWM. The potential uncertainty related to this type of flow disturbance has been investigated with acoustic Doppler current profilers, which measure from the water surface vertically downward to the channel bottom
(Mueller et al. 2007; Muste et al. 2010). The flow disturbance caused by the ADVM mounted on the channel bottom could result in a decrease or increase in the ADVM velocity measurements. From the laboratory testing, the bias was toward overestimation of the cross-sectional average velocity by the VCWM, which could indicate that the ADVM disturbance resulted in an increase in velocity measurements. However, this overestimation in crosssectional average velocity should have decreased for the largest buffer distance because measurements were taken further away from the disturbance, but this was not the case. Further research in ADVM disturbance and appropriate buffer distances for specific ADVM designs is warranted.

Typically, an upward-looking ADVM takes samples every second and the average of the velocities at each measurement depth are collected and stored over intervals of 5-15 min (considered real time) depending on user settings. It is important to note that the ADVM must be set up to store point velocity measurements so that the VCWM can be applied. Occasionally, albeit infrequently, a high velocity point could be recorded by the ADVM at a depth that is relatively low in the flow profile (below $50 \%$ of the flow depth). The VCWM assumes that the high velocity region will be above $50 \%$ of the flow height, therefore the maximum velocity depth should be limited to this range. It is not necessary to remove the high velocity measurement below $50 \%$ of the flow height from the Eq. (6). This anomalous velocity should not be appreciably out of range of neighboring measurements, and the weight associated with any single measurement is relatively small resulting in insignificant errors. However, the location of the maximum velocity point, $z_{U \text { max }}$, is essential for the formation of the weighting function and should be located in the upper $50 \%$ of the velocity profile as noted by Chow (1959).

The VCWM is capable of computing weights and $U_{1}$ on a realtime basis as the water depth, velocity, and location of the high velocity region change relatively slowly over timescales of minutes to tens of minutes. Because of the variability in velocities measured every second, which are likely attributed to turbulence and acoustic noise, the VCWM will be most effective when applied to the velocities measured at each depth that have been averaged over the user supplied interval (e.g., 5-15 min intervals). The VCWM equations are straightforward and robust, which make them well suited for these real-time deployments. Relatively small channel scenarios were used to develop the VCWM. Future testing is needed in larger channels with bottom widths greater than $1.5 \mathrm{~m}$ and water depths greater than $1 \mathrm{~m}$ to verify VCWM effectiveness.

\section{Summary and Conclusions}

A new method is developed to estimate the cross-sectional average velocity in an irrigation channel using an upward-looking ADVM. The Velocity Contour Weighting Method (VCWM) computes the average velocity as a weighted average of bin velocities measured by the ADVM, where weights depend on the channel geometry, water depth, and the location of the maximum velocity. Computational fluid dynamics (CFD) is used to develop a detailed set of data on the cross-sectional distribution of streamwise velocity, covering a range of channel and flow configurations. A regression technique is then used to develop predictive equations for the velocity weights. A second procedure is developed to estimate the buffer region velocity where the upward-looking ADVM cannot measure. A power-law is used to model the buffer region velocity, and CFD data are again used to develop predictive equations for the powerlaw parameters. 
Laboratory testing shows that the VCWM can be used to estimate discharge with uncertainty less than $\pm 5 \%$ without calibration. This is an improvement on the $\pm 6 \%$ uncertainty with the conventional index-velocity method in a uniform cross section with a recommended 10 calibration points (Styles et al. 2006). The best strategy to minimize this error is to limit the buffer distance near the channel boundary provided that the ADVM interference on the velocity distribution can be minimized.

VCWM offers several advantages over the commonly used index-velocity method. Leveraging the velocity distribution measured by the upward-looking ADVM, the VCWM algorithm breaks out the independent components of channel geometry, water depths, and surface roughness to circumvent the need for the intensive index-velocity calibration process under varying channel conditions. Channel geometry can be measured by surveying the site, water depth by the ADVM, and surface roughness can be estimated by using tables in most hydraulics textbooks. The sensitivity to surface roughness parameter is examined in a companion paper.

\section{Acknowledgments}

The writers would like to acknowledge Dr. Charles Burt, Chairman, Irrigation Training and Research Center at California Polytechnic State University, San Luis Obispo, for access to the Cal Poly ITRC flume and Mohamed Grissa for his participation in the laboratory experiments. This research was made possible by a grant from the University of California Prosser Trust (PR-020), whose support is gratefully acknowledged.

\section{Notation}

The following symbols are used in this paper:

$A=$ hydraulic or wetted cross-sectional area;

$A_{i}=$ area within velocity contours;

$a=$ power-law coefficient based on power-law in Eq. (9);

$C_{a}=$ coefficient for a derived as $a$ function of $z_{b}$;

$c=$ coefficient for shear velocity power-law;

$\mathrm{F}=$ Froude number;

$h=$ flow depth;

$k_{s}=$ equivalent roughness height;

$m=$ power-law exponent;

$R_{h}=$ hydraulic radius;

$R_{s}=$ channel shape factor;

$S S=$ channel side slope;

$U_{i}=$ velocity sampled by the upward-looking ADVM at $z_{i}$;

$U_{\max }=$ maximum velocity within a grid of individual velocity samples;

$U_{1}=$ average velocity in the buffer region;

$u_{i}=$ velocity at contour boundary;

$u_{*}=$ shear velocity;

$V=$ cross-sectional average velocity;

$V_{\mathrm{ADVM}}=$ depth-averaged velocity from the actual upwardlooking ADVM;

$V_{\mathrm{VCWM}}=$ calculated cross-sectional average velocity using VCWM;

$w_{i}=$ weight of velocity $U_{i}$;

$w_{1}=$ weight within buffer region;

$z=$ normal distance from channel bottom;

$z^{\prime}=$ characteristic length;

$z_{b}=$ buffer distance determined as the height from the channel bottom to the first ADVM sample; $z_{b}^{\prime}=$ distance to first velocity contour $\left[z_{b}^{\prime}=z_{b}-0.5\right.$ $(0.034 \mathrm{~m})]$;

$z_{U \max }=$ height at the maximum velocity point;

$\Delta z=$ vertical distance between ADVM velocity samples; and $\kappa=$ von Kármán constant.

\section{References}

ASCE Task Force on Friction Factors in Open Channels (1963). "Friction factors in open channels." J. Hydraul. Div., 89(2), 97-143.

Chen, C. L. (1991). "Unified theory on power laws for flow resistance." J. Hydraul. Eng., 117(3), 371-389.

Cheng, N.-S. (2007). "Power-law index for velocity profiles in open channel flows." Adv. Water Resour., 30(8), 1775-1784.

Chow, V. T. (1959). Open-channel hydraulics, McGraw-Hill, New York.

Cook, C. B., and Richmond, M. C. (2001). "Simulation of tailrace hydrodynamics using computation fluid dynamics models." PNNL-13467, Pacific Northwest National Laboratory, Richland, WA.

Gupta, R. S. (1989). Hydrology and hydraulic systems, Prentice Hall, Englewood Cliffs, NJ.

Hardcastle, P. J., and Thorne, P. D. (1997). "Simultaneous measurements of high resolution vertical and horizontal flow profiles and suspended sediments using acoustic backscattering." 7th Int. Conf. on Electronic Engineering in Oceanography, IEE, Southampton, UK, 100-106.

Hirt, C. W., and Nichols, B. D. (1981). "Volume of fluid (VOF) method for the dynamics of free boundaries." J. Comput. Phys., 39(1), 201-225.

Howes, D. J., Burt, C. M., and Sanders, B. F. (2010). "Subcritical contraction for improved open channel flow measurement accuracy with an upward-looking ADVM.” J. Irrig. Drain Eng., 136(9), 617-626.

Huhta, C., and Ward, C. (2003). Flow measurements using an upwardlooking argonaut-SW Doppler current meter, IEEE, San Diego, 35-39.

Keulegan, G. H. (1938). "Laws of turbulent flow in open channels." J. Res. Natl. Bur. Stand., 21(6), 707-741.

Maghrebi, M. F., and Rahimpour, M. (2005). "A simple model for estimation of dimensionless isovel contours in open channels." Flow Meas. Instrum., 16(6), 347-352.

Montes, S. (1998). Hydraulics of open channel flow, ASCE, Reston, VA.

Morlock, S. E., Nguyen, H. T., and Ross, J. H. (2002). Feasibility of acoustic doppler velocity meters for the production of discharge records from, U.S. Geological Survey streamflow-gaging stations, U.S. Geological Survey, Indianapolis.

Mueller, D. S., Abad, J. D., Garcia, C. M., Gartner, J. W., Garcia, M. H., and Oberg, K. A. (2007). "Errors in acoustic Doppler Profiler velocity measurements caused by flow disturbance." J. Hydraul. Eng., 133(12), $1411-1420$

Muste, M., Kim, D., and Gonzalez-Castro, J. A. (2010). "Near-transducer errors in ADCP measurements: Experimental findings." J. Hydraul. Eng., 136(5), 275-289.

Patino, E., and Ockerman, D. (1997). "Computation of mean velocity in open channels using acoustic velocity meters." Open File Rep. 97-220, U.S. Geological Survey, Tallahassee, FL.

Replogle, J. A. (1997). "Practical technologies for irrigation flow control and measurement." Irrig. Drain. Syst., 11(3), 241-259.

Replogle, J. A., and Kruse, E. G. (2007). "Delivery and distribution systems." Design and operation of farm irrigation systems, G. J. Hoffman, R. G. Evans, M. E. Jensen, D. L. Martin, and R. L. Elliott, eds., American Society of Agricultural and Biological Engineers (ASABE), St. Joseph, MI, 347-391.

Simpson, M. R. (2001). "Discharge measurements using a broad-band acoustic Doppler Current Profiler." Open-File Rep. 01-1, U.S. Geological Survey, Sacramento, CA.

Styles, S. W., Busch, B., Howes, D., and Cardenas, M. (2006). "Nonstandard structure flow measurement evaluation using the flow rate indexing procedure-QIP.” ITRC Rep. No. R 06-003, Irrigation Training and Research Center (ITRC), California Polytechnic State Univ., San Luis Obispo, CA. 